\title{
The Influence of External Pressure on Resonant Frequency of SAW Resonator
}

\author{
K. JASEK AND M. PASTERNAK* \\ Military University of Technology, S. Kaliskiego 2, 00-490 Warsaw, Poland \\ (Received May 12, 2014)
}

\begin{abstract}
The results of numerical simulations and experimental measurements of surface acoustic wave resonator centre frequency and amplitude dependence on external pressure changes in the range $0-1$ atm are presented in the paper. According to the analytical model as well as numerical calculations the pressure influence on the resonator centre frequency is linear. Unfortunately, in the real measurements the linearity is difficult to observe. Additional effects that modify the linear dependence of the centre frequency on pressure are caused by water particles. It is well visible especially in the low pressure range. The phenomenon can be applied for research of water contents in dry gases as well as construction of acoustoelectronic vacuometers.
\end{abstract}

DOI: $10.12693 /$ APhysPolA.127.1601

PACS: 02.60.- $\mathrm{x}$, 43.35.Pt, 46.80. $+\mathrm{j}, 61.30 . \mathrm{Hn}$

\section{Introduction}

The operation of the surface acoustic wave (SAW) sensors is based on measurement of its resonance frequency change caused by physical interactions between certain environment factors and SAW substrate. In this type of sensors fluctuations of external gas pressure and temperature play an important role. The reason for this is not only the ideal gas law but also the influence of the parameters of immediate surroundings on the SAW propagation conditions. That is why these parameters have to be stabilised during laboratory measurements.

The temperature influence was investigated for many years and it is described in detail in many papers (e.g. $[1,2])$. Based on this influence the SAW temperature sensors are constructed [3]. The influence of external pressure on the SAW substrate is also investigated in the context of measurement [4]. In most cases the research is focused on the changes of SAW parameters under high pressure when mechanical stress (mass) effect dominates.

The measurement results presented in the paper show that the low-pressure range is also very interesting from the point of view of applications. In the range stiffening effect of adsorbed water particles starts to be significant. Because the stiffening effect overlap the mass effect the change of SAW device centre frequency with pressure becomes more compound and nonlinear in particular. For pressures close to 0 the frequency changes are again positive and linear.

The results of simulations as well as experimental measurements of pressure influence on SAW resonator centre frequency and amplitude for low pressures are presented in the paper.

*corresponding author; e-mail: mpasternak@wat.edu.pl

\section{Theoretical background}

The SAW velocity and amplitude can change with the change of environment parameters. On this fact the operational principle of SAW sensors is based; the change in velocity is usually transformed to the change of centre frequency of the devices. To amplify the effect, different method to increase surface sensitivity are applied, starting from special surface preparation up to sensitive layers deposition. The surface even without special treatment is sensitive to many physical parameters. For example, according to research based on numerical experiments it is sensitive to external pressure change and such dependence is observed for each of the excited modes. The deformations for one of them in the two-port SAW resonator are presented in Fig. 1.

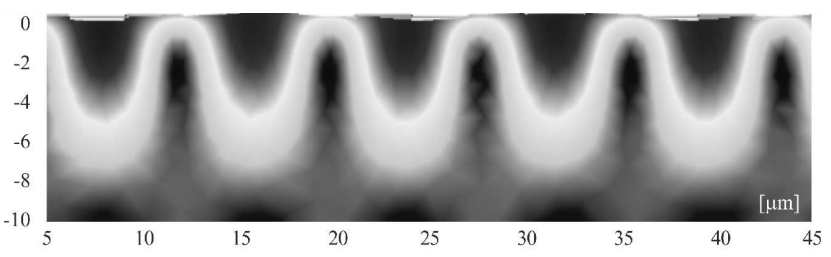

Fig. 1. The deformations for a chosen mode of the surface fragment of two-port SAW resonator under low external pressure.

However, the calculations taking into consideration only mechanical interaction show that the pressure variations cause very small and negative centre frequency changes of order of $-1 \mathrm{ppm} \mathrm{m} \mathrm{m}^{2} / \mathrm{g}$. On the other hand, the changes observed in a real measurements far exceed the expectation based on theoretical model investigation. Moreover, for the pressures close to 0 their character changes from positive to negative. The comparison of an exemplary simulation and measurement results is shown in Fig. 2.

The main reason of the striking discrepancy visible in the example in Fig. 2 can be relevant with the humidity. 


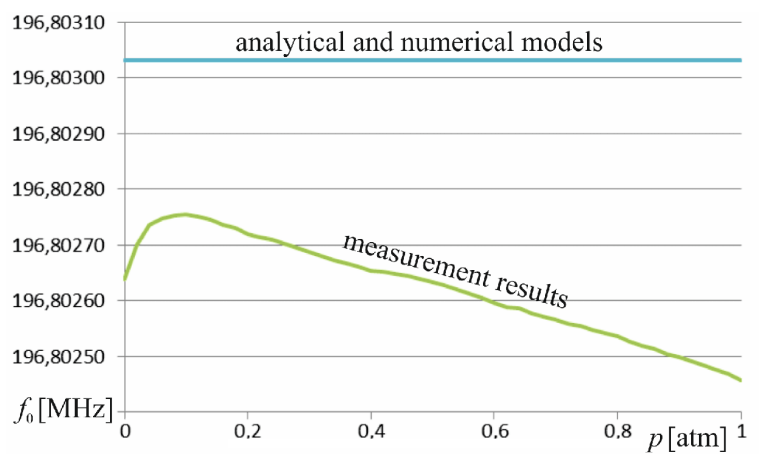

Fig. 2. Comparison of exemplary simulation and measurement results. The theoretical changes are far smaller than these observed in measurements.

In theoretical models the pressure influence is often described in terms of mechanical mass surface loading without taking any other phenomena into consideration. Actually in such kind of sensors that inherently are exposed to real environment factors at least three kinds of effects have to be considered. The effects have additive character and can be described using the formula $[5,6]$ :

$$
\begin{gathered}
\Delta f=-C_{\mathrm{m}} f_{0}^{2} \rho_{\mathrm{S}}+4 C_{\mathrm{e}} \frac{f_{0}^{2}}{v_{0}^{2}} h G_{\mathrm{S}} \\
-f_{0} \frac{K^{2}}{2} \frac{\sigma_{\mathrm{S}}^{2}}{\sigma_{\mathrm{S}}^{2}+\left(v_{0} C_{\mathrm{S}}\right)^{2}},
\end{gathered}
$$

where $C_{\mathrm{m}}$ and $C_{\mathrm{e}}$ describe the coefficients of mass sensitivity and elasticity of the substrate, respectively, $\rho_{\mathrm{S}}$ is the surface density of the deposited layer (here water), $f$ - centre frequency of the SAW sensor, $v$ - phase velocity of unperturbed Rayleigh wave, $h$ - the layer thickness, $G_{\mathrm{S}}$ - shear modulus of the layer, $K^{2}$ - the electromechanical coupling coefficient, $\sigma_{\mathrm{S}}=\sigma h$ is the layer conductivity, where $\sigma$ represents the bulk conductivity of layer material, $C_{\mathrm{S}}$ describes the capacitance per unit length of the SAW substrate material; $C_{\mathrm{S}}=\varepsilon_{\mathrm{S}}+\varepsilon$, where $\varepsilon_{\mathrm{S}}$ and $\varepsilon$ are the permittivities of the substrate and free space, respectively.

The first negative term of Eq. (1) represents mass effect and is usually taken into consideration for pressure influence modelling. The quantitative analysis of this term show that in an average conditions it is very small. For instance, for quartz ST substrate at $100 \mathrm{MHz}$ $C_{\mathrm{m}} \approx 1.29 \times 10^{-10} \mathrm{~m}^{2} \mathrm{~s} / \mathrm{g}$, that gives the changes in order of $-1 \mathrm{ppm} \mathrm{m} \mathrm{m}^{2} / \mathrm{g}$. Very similar values were obtained from numerical simulations neglecting the presence of water.

The second term is positive and describes the stiffness effect of the surface caused by layer existence. According to the calculations carried out for water layer this term can be much bigger than the first one and increases when the layer thickness decreases. It is the effect related to the water surface tension phenomenon.

The last term, again negative, expresses the layer electric conductivity influence. The dependence of relative frequency change vs. relative surface conductivity is shown in Fig. 3.

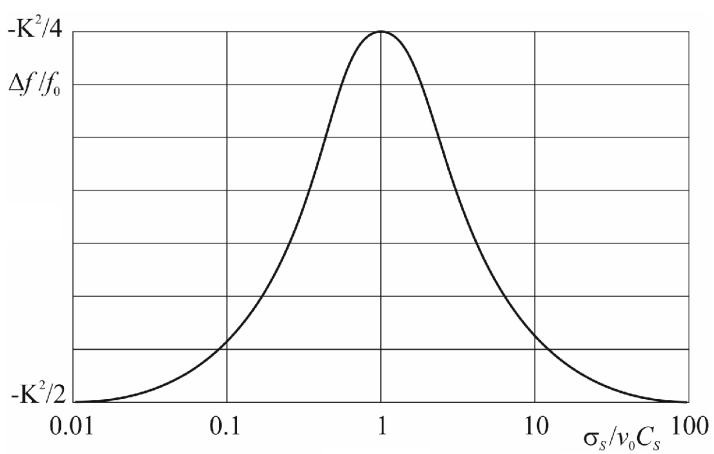

Fig. 3. Relative frequency change vs. relative surface conductivity.

According to the function illustrated in Fig. 3, extreme values of conductivity are ineffective for centre frequency changes [7]. Close to the electrically shorted surface condition the surface stiffness aims at constant value and close to open one it vanishes. The water particles layer fulfil rather open conditions because the conductivity of pure water layer is very small and depends on the number of water monolayers deposited on a substrate [8]. The dependence of conductivity and number of water monolayers on relative ambient humidity for quartz surface is illustrated in Fig. 4. It is worth to note that bulk conductivity of ultrapure water at $25^{\circ} \mathrm{C}$ equals about $5.5 \times 10^{-6} \mathrm{~S} / \mathrm{m}$ due to hydrogen and hydroxide ions existence.

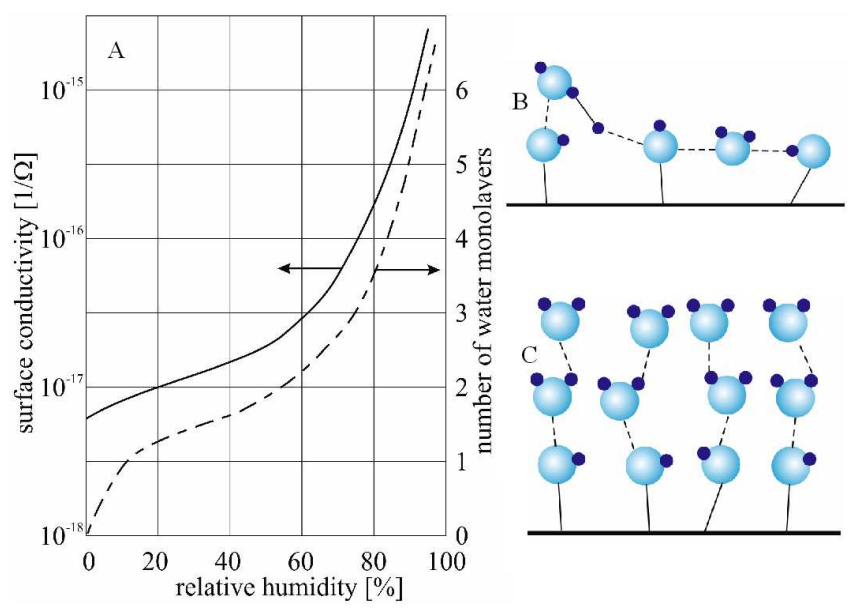

Fig. 4. Surface conductivity and number of water monolayers vs. relative humidity [8] (A), water on quartz surface in vacuum (B), physically adsorbed water at $25^{\circ} \mathrm{C}$.

The hydrogen bonds, a dipole-dipole attraction between water and substrate particles, are particularly strong. For this reason the water monolayer exists even in the vacuum conditions and special procedures are needed for its total removal.

The short analysis presented above shows that two effects connected with water presence could explain the 
measurement results. First one, close to vacuum, is induced by water surface tension that increases surface stiffness and the second, for higher pressures, is the mass effect induced by the adsorption of water molecules. The two effects are much more stronger than the stress effect induced by pressure. Because of different character there is a kind of interaction between them and for higher pressures the mass loading effect dominates. That is why the results from measurement are nonlinear and differ from simulation taking into consideration only the mechanical stress interaction. This conclusion was verified by the measurements.

\section{Measurement results}

The measurements were carried on using two different experimental setups. The first one was modified to measure SAW resonator frequency changes versus atmospheric pressure variation typical for the SAW sensors operation. In the measurements a two-port SAW resonator was used. The resonator as well as oscillator circuits have been described in $[9,10]$. The schematic view of the setup is shown in Fig. 5.

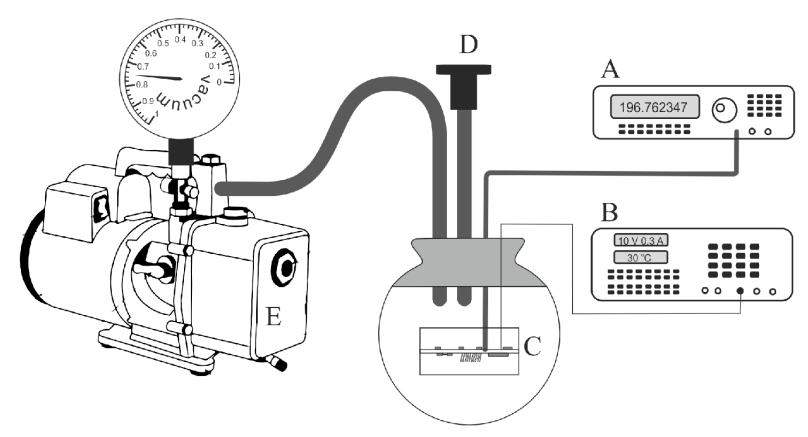

Fig. 5. Setup for measurements in the natural air environment: A - frequency meter, B - power supply and temperature measurement, $\mathrm{C}$ - hermetic chamber with a SAW stabilised oscillator, D - air valve, E vacuum pump with vacuometer.

In the second experimental setup nitrogen with known water content was used with molecular sieves that allowed to decrease the humidity down to $0.23 \mathrm{ppm}$. Beside frequency changes the second setup enable to measure SAW amplitude attenuation. This setup has been described in detail in [11]. It consists of external ultrastable SAW excitation unit and digital output signal analyser. It is worth to stress that the measurement methods for both setups are different and that the measurements are independent. The exemplary measurement results obtained using the first setup are shown in Fig. 6.

The value of $0 \mathrm{~atm}$ in Fig. 6 (and all subsequent) corresponds to vacuum in the medium level of $10^{-3}$ Torr. During all measurements the temperature was constant with accuracy $\pm 1{ }^{\circ} \mathrm{C}$.

The presumption that the key role in the shaping of the dependence presented here is played by water particles

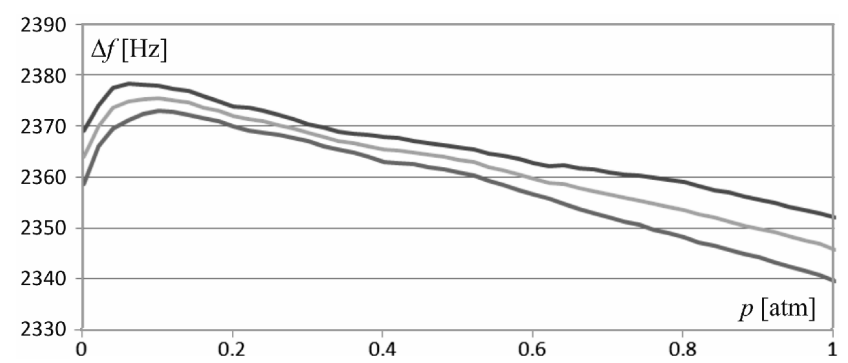

Fig. 6. SAW resonator frequency change vs. external pressure. Tree different series of measurements in the same setup are presented. The measurements give reproducible results.

comes from the observation that the frequencies increase when the SAW resonator was kept longer in vacuum maintained by a vacuum pump. Since the observation is repeatable, so the characteristic must be determined by water adsorption/desorption processes. The examples of typical characteristics obtained while decreasing pressure and increasing after long conditioning in vacuum are presented in Fig. 7.

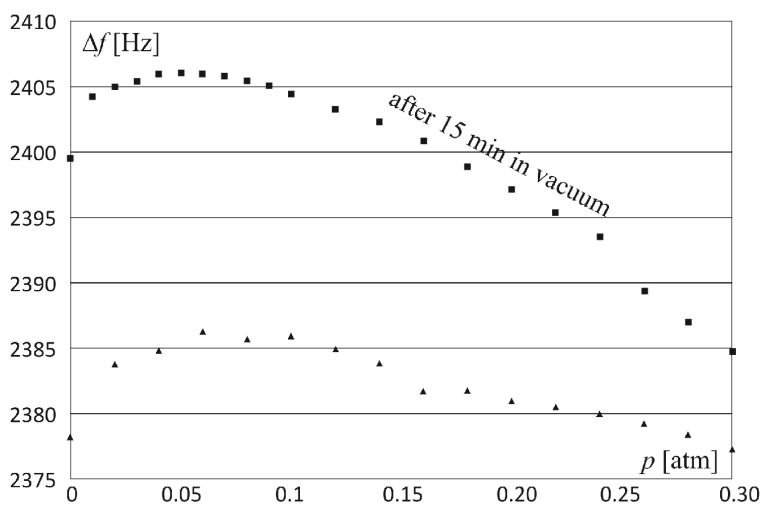

Fig. 7. Exemplary measurement results measured frequency change vs. pressure. The lower curve was obtained during the pressure decreasing and the upper during pressure increasing after 15 min detaining of SAW resonator in the vacuum.

The slope of the upper curve in Fig. 7 is somewhat bigger than lower one and the curves reach similar frequencies at the normal pressure. The fact allows to conclude that the adsorption process is insignificantly slowing down as the number of adsorbed water particles increases.

The presumptions were confirmed by the results of measurements carried out on using second setup with and without molecular sieves. The time of vacuum detaining was in this case lengthened to $30 \mathrm{~min}$. The exemplary results of measurements are shown in Fig. 8.

As the adsorption and desorption processes are energetically different, some hysteresis is observed in Fig. 8. It is interesting to note that SAW is sensitive to such small amount of water like $0.23 \mathrm{ppm}$. The SAW sensitivity to humidity at normal pressure was investigated 
in [12] and it was shown that for the ultra-dry environment the frequency changes are very small and correspond to the results of theoretical simulations. In such kind of environment both the stiffness and mass effects are insignificant.

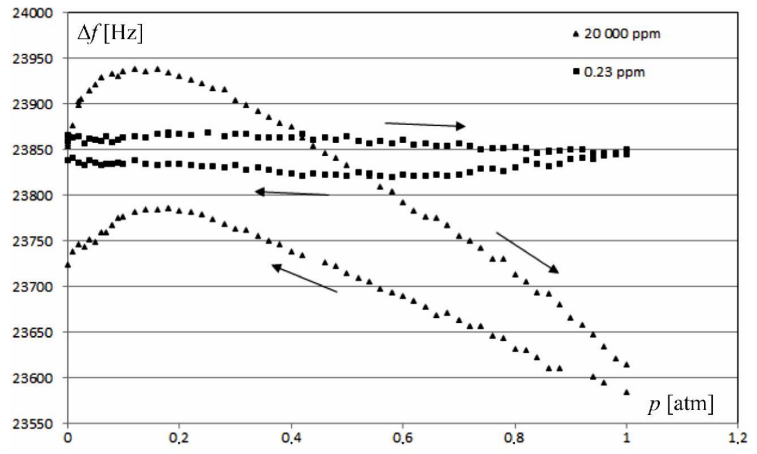

Fig. 8. The frequency changes vs. pressure for humid environment (triangles $-20000 \mathrm{ppm}$ of water) and dry one (squares - $0.23 \mathrm{ppm}$ of water). Arrows indicate the direction of pressure change.

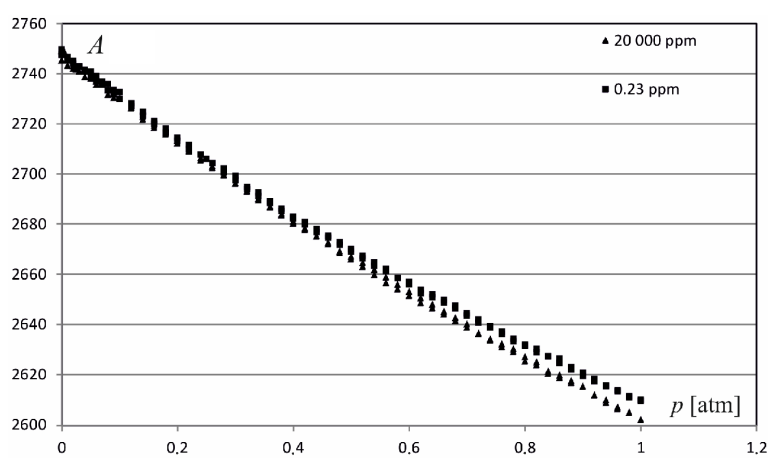

Fig. 9. Attenuation vs. pressure for dry and humid environment. The attenuation is given here in arbitrary units.

The second measurement setup allowed for the measurement of the SAW attenuation. The exemplary results are shown in Fig. 9.

No visible hysteresis is noticeable in Fig. 9 but close to normal pressure the curves for humid and dry atmosphere split. The results allow us to conclude that the water adsorption does not affect significantly the resonator attenuation in low-pressure condition. In this case pressure plays the key role. However close to the normal pressure the influence of the mass of the adsorbed water is noticeable.

\section{Conclusions}

The measurements of the SAW resonator centre frequency changes with pressure from medium vacuum to normal pressure allow to conclude that there are two main mechanisms responsible for the changes. The first one is positive and dominates for lower pressure values; for quartz substrate it is observed below $0.1 \mathrm{~atm}$.
The effect is induced by water surface tension. When the pressure increases and the adsorbed water layers number grows the dominant role is played by the water particles mass loading. Since this effect is negative it causes the bend of the frequency-pressure characteristics in the lower pressure range. The apex of the characteristic indicates the equilibrium point between the two mentioned effects. The presence of water almost does not affect the SAW attenuation in the low-pressure range. In this range the parameter vary with pressure and the dependence is linear. On can say that in SAW devices response lowpressure variation is better represented by attenuation than frequency changes.

Because the stiffness effect is linear close to vacuum it can be utilized to construct a new kind of vacuometer. The phenomena observed during the measurements described above can be applied to investigate physical properties of water monolayers as well as water content level in technical and scientific gases, for example.

\section{References}

[1] C.S. Lam, in: Proc. 4th Int. Symp. on Acoustic Wave Device for Future Communication Systems, Chiba 2010

[2] A.B. Randles, J.M. Tsai, P. Kropelnicki, H. Cai, J. Automat. Control Eng. 2, 191 (2014).

[3] Y.H. Kim, D.H. Chang, Y.S. Yoon, J. Korean Phys. Soc. 45, 1366 (2004).

[4] J.G. Rodríguez-Madrida, G.F. Iriartea, O.A. Williams, F. Calle, Sensors Actuat. A 2, 364 (2013).

[5] A.J. Ricco, S.J. Martin, Thin Solid Films 206, 94 (1991).

[6] M. Penza, P. Aversa, G. Cassano, W. Wlodarski, K. Kalantar-Zadeh, Sensors Actuat. B 127, 168 (2007).

[7] M. Urbańczyk, W. Jakubik, J. Techn. Phys. 38, 589 (1997).

[8] Y. Awakuni, J.H. Calderwood, J. Phys. D Appl. Phys. 5, 1038 (1972).

[9] K. Jasek, S. Neffe, M. Pasternak, Acta Phys. Pol. A 122, 825 (2012).

[10] W. Soluch, IEEE Trans. Ultrason. Ferroelect. Freq. Contr. 55, 2519 (2008).

[11] K. Jasek, W. Miluski, M. Pasternak, Acta Phys. Pol. A 124, 445 (2013).

[12] K. Jasek, M. Pasternak, Acta Phys. Pol. A 124, 448 (2013). 